\title{
Antimicrobial therapy for the treatment of opportunistic infections in HIVIAIDS patients: a critical appraisal
}

This article was published in the following Dove Press journal:

HIVIAIDS - Research and Palliative Care

I April 201 I

Number of times this article has been viewed

\author{
Jo Seddon' \\ Sanjay Bhagani ${ }^{2}$ \\ 'Department of Infectious Diseases, \\ Imperial College Healthcare NHS \\ Trust, London, UK; ${ }^{2}$ Department of \\ Infection and Immunity, Royal Free \\ Hospital NHS Trust, London, UK
}

Correspondence: Jo Seddon

Department of Infectious Diseases, 8th floor Commonwealth Building, Hammersmith Campus, Du Cane Road, London WI2 OHS, UK

Tel +442033l3 I000

Email joseddon@nhs.net
Abstract: The widespread use of antiretroviral therapy (ART) has entirely changed the management of human immunodeficiency virus (HIV) infection and dramatically reduced the rates of opportunistic infections (OI). However, OI continue to cause significant morbidity and mortality in both developed countries, where presentation with advanced HIV infection is common, and also in developing countries where ART is less widely available. Evidence to direct OI guidelines is partly limited by the fact that many large-scale studies date from the pre-ART era and more recent studies are sometimes poorly powered due to the falling rates of OI. Treatment of OI is now known to be as much about antimicrobials as about immune reconstitution with ART, and recent studies help guide the timing of initiation of ART in different infections. OI have also become complicated by the immune reconstitution inflammatory syndrome phenomenon which may occur once successful immune recovery begins. Trimethoprim-sulfamethoxazole has long been one of the most important antibiotics in the treatment and prevention of OI and remains paramount. It has a broad spectrum of activity against Pneumocystis jiroveci, toxoplasmosis, and bacterial infections and has an important role to play in preventing life-threatening OI. New advances in treating OI are coming from a variety of quarters: in cytomegalovirus eye disease, the use of oral rather than intravenous drugs is changing the face of therapy; in cryptococcal meningitis, improved drug formulations and combination therapy is improving clearance rates and reducing drug toxicities; and in gut disease, the possibility of rapid immune restitution with ART is replacing the need for antimicrobials against cryptosporidia and microsporidia.

Keywords: HIV, AIDS, opportunistic infections, treatment, antimicrobials

\section{Introduction}

Advances in the treatment of human immunodeficiency virus (HIV) with effective antiretroviral therapy (ART) in the last 20 years have dramatically changed the face of HIV infection. This has led to a sharp reduction in both opportunistic infections (OI) and death rates. In spite of this, OI continue to cause significant morbidity and mortality in patients with HIV all around the world. In developed countries, late presentation of HIV remains a considerable problem, with over $20 \%$ of patients in the UK presenting with CD4 $<200$ cells $/ \mu \mathrm{L}$ and therefore being at risk of OI. ${ }^{1}$ Other patients have a suboptimal response to ART due to poor adherence to therapy, drug toxicities, and extensively drug-resistant HIV virus, so remain immunosuppressed in spite of treatment. In developing countries, lack of access to ART and medical facilities makes OI a common occurrence.

A critical review of antimicrobial treatment for OI is necessary because while the OI themselves remain fairly static over time, the number of agents that are now 
available for the treatment of specific OI is ever increasing and in some cases the incidence of drug resistance forces a change in treatment guidance. Moreover, the use of ART in addition to, and in some occasions instead of, antimicrobials has altered both the drugs used and their treatment duration. Thus, a review of antimicrobial treatment of OI now that the use of ART is widely established brings together new knowledge from the ART-era as well as using many studies performed before the widespread use of ART.

However, it is a limitation of this review that many of the large-scale randomized control studies quoted were carried out prior to the widespread availability of effective ART and a consequence of the decline in incidence of OI is that many centers no longer see a large enough number of specific infections to carry out well-powered controlled studies. This appraisal does not seek to be a comprehensive review of the treatment of all OI, but more a discussion on the data that guides everyday practice in treating the majority of infective admissions in HIV-infected individuals in the developed world.

\section{Methods}

This review was carried out by performing a PubMed database search on the various topics OI covers. It included studies from both the pre- and the post-ART era and this is highlighted in the text where relevant. Due to the recent nature of the disease, all studies that relate specifically to HIV date from the mid-1980s onwards.

\section{The role of ART}

The majority of patients who present with OI are not already taking ART, therefore initiation of ART and the specific timing of ART initiation will play a major role in the management of the acute OI. For certain OI where there is no specific antimicrobial treatment, ART may be the only treatment available; in other infections, patients will be stabilized with antimicrobials before starting ART. Recent data regarding the specific timing of introducing ART generally favors early initiation ${ }^{2,3}$ but this must always be balanced with the problem of overlapping drug toxicities, drug-drug interactions and the potential for an immune reconstitution inflammatory syndrome (IRIS) to complicate the management of the acute OI.

The data that guides the introduction of ART during an acute OI comes from several studies conducted in different parts of the word. The AIDS Clinical Trial Group (ACTG) carried out a large randomized trial where patients either started ART "early" ie, within 14 days of starting OI treatment, or "late" ie, after completion of OI treatment. Importantly, patients with tuberculosis (TB) were excluded from the study, but they demonstrated both fewer deaths and less AIDS progression in the early initiation arm. ${ }^{3}$ The SAPIT study in Durban, South Africa looked specifically at TB and randomized patients into "integrated treatment" and "sequential treatment" arms depending on when ART was initiated. They found that the integrated treatment arm, which started ART within the first 3 months of TB treatment, had a $56 \%$ relative reduction in mortality compared to those who started after TB treatment completion. ${ }^{2}$ A further study performed in Cambodia, the CAMELIA trial, found a similar benefit in early use of ART in conjunction with TB treatment. ${ }^{4}$ There have been worries that starting ART early increases the risk of IRIS, however, a review of the data from the ACTG study has not shown that early initiation of ART is associated with a greater risk of IRIS. They did however find that in patients in advanced immunosuppression, risk factors for IRIS included: fungal infection, lower CD4+ T-cell counts and higher HIV RNA levels at baseline, and higher CD4+ T-cell counts and lower HIV RNA levels on treatment. ${ }^{5}$

\section{Overview of antimicrobial therapy in HIV}

Probably the most widely used antibiotic worldwide in the HIV-infected population is trimethoprim-sulfamethoxazole (TMP-SMX) which is used as prophylaxis for Pneumocystis jiroveci pneumonia (PCP) in patients with CD $4<200$ cells $/ \mu \mathrm{L}$. Its additional prophylactic activity against toxoplasmosis and its antibacterial activity against a number of important bacterial pathogens including Salmonella spp. and Streptococcus pneumoniae has led to a general decrease in the number of life-threatening bacterial infections occurring in patients with advanced HIV infection, particularly in resource-poor settings, although the rates have not decreased to those seen in the HIV-negative population. TMP-SMX is a relatively broad-spectrum antibiotic that has activity against many other common respiratory, skin, and bowel pathogens. Despite the widespread use of TMP-SMX worldwide, resistance to PCP is not commonly reported and most cases of PCP and toxoplasmosis respond to first-line agents. ${ }^{6,7}$

\section{Respiratory infections}

Respiratory symptoms may be caused by a number of different OI, particularly given that HIV-associated T and B cell dysfunction increases the incidence of respiratory infections at all CD4 counts. PCP remains the most common and clinically important severe OI, although bacterial infections, 
Mycobacterium tuberculosis and other fungal and viral infections should always be considered. In general, a clinician is able to rapidly risk assess a patient depending on the presenting symptoms and the degree of immunosuppression. This is important, as it is almost always necessary to commence antimicrobial therapy before laboratory confirmation or isolation of the suspected pathogen. A notable exception to this rule may be mycobacterial infections, which is beyond the scope of this review article.

\section{Pneumocystis jiroveci pneumonia}

Pneumocystis jiroveci pneumonia (PCP) is caused by the fungus Pneumocystis jiroveci, a ubiquitous organism that usually causes primary infection in early childhood. Current evidence is in favor of disease in the immunosuppressed being caused largely by reinfection with the fungus although reactivation of latent infection may also occur. ${ }^{8}$ PCP often has an insidious onset and patients classically present with dry cough and shortness of breath on exertion.

Any consideration of antimicrobials for the treatment of PCP must take several factors into consideration:

1. the severity of disease, as determined by hypoxemia (ie, $\mathrm{PaO} 2<9.3 \mathrm{kPa}$ or $\mathrm{SpO} 2<92 \%$ on room air)

2 . the need for intravenous versus oral medication

3. the tolerability of the regime and the impact of drug toxicities

4. prior treatment with PCP prophylaxis.

\section{First-line treatment for severe PCP}

Most clinicians agree that first-line treatment for severe disease remains with high-dose intravenous TMP-SMX for 21 days. It has an efficacy of around $90 \%$ in mild disease and $70 \%$ in severe disease. This has been compared extensively against other regimes including pentamidine, primaquine and clindamycin, atovaquone, and dapsone. The only other drug that has similar efficacy in severe PCP is intravenous pentamidine.

The initial evidence for the treatment of PCP came from a number of studies performed in the pre-ART era. ${ }^{6}$ The largest of these studies, published in 1992, enrolled 163 patients and compared high dose intravenous TMP-SMX (120 mg/kg/day) with intravenous pentamidine ( $4 \mathrm{mg} / \mathrm{kg} /$ day). In general, these two agents compared equally in terms of efficacy and toxicity. ${ }^{9}$ In other comparative studies, side effects in both treatment arms were nearly universal and failure to complete treatment or the need to switch regimen was often as high as $40 \%$. The most common side effects of TMP-SMX were rash and anemia, and of pentamidine were nephrotoxicity, hypotension, and hypoglycemia.
Supportive care for the side effects of TMP-SMX may be effective, and persisting by "treating through" the mild side effects can limit the need for a treatment change. ${ }^{10,11}$ This is not the case with pentamidine where nephrotoxicity normally necessitates switching treatment. For this reason, TMP-SMX has remained the first-line treatment for PCP in OI guidelines from both the British HIV Association, which are due to be published in 2011, and the Infectious Diseases Society of America (IDSA). ${ }^{12}$ Ultimately, the decision to treat with one drug or another is often made on an individual patient basis, depending on side effect profile. It should be added that there have been no recent well-powered studies comparing the two agents head-to-head. Prior treatment with TMP-SMX prophylaxis is not a contra-indication to its use at treatment dose as PCP infection is normally a result of poor adherence or drug absorption rather than drug resistance.

Patients with severe PCP, as defined by $\mathrm{PaO} 2<9.3 \mathrm{kPa}$ or $\mathrm{SpO} 2<92 \%$ on room air, should receive corticosteroids, and a survival benefit has been clearly demonstrated in a number of studies. ${ }^{13,14}$ The preferred regimen of prednisolone is $40 \mathrm{mg}$ twice daily for days $1-5,40 \mathrm{mg}$ daily for days 5-10, and $20 \mathrm{mg}$ daily for days 11-21. If intravenous steroids are required, then intravenous methylprednisolone given at $75 \%$ of the prednisolone doses is used. ${ }^{15}$

\section{Second-line treatment for PCP}

Robust data exists regarding second-line treatment regimes and treatment for mild to moderately severe PCP and there are a number of different reasonable treatment options that may be used according to an individual clinician's preference. A study by Safrin et al compared three oral regimens in nonsevere PCP: TMP-SMX, dapsonetrimethoprim, and clindamycin-primaquine. 181 patients were randomized to one of the three treatment arms and the study concluded that there were no statistically significant differences between the groups in terms of the rates of therapeutic failure, drug toxicities, or completion of the treatment course. ${ }^{10}$ Other studies have shown similar results, including a double-blind randomized multicenter comparison of TMP-SMX with clindamycin-primaquine which reported a treatment success rate of $79 \%$ and $76 \%$, respectively. Atovaquone has also been used in the treatment of PCP, and is reported to be less efficacious but better tolerated when compared to TMP-SMX. ${ }^{16}$ Ultimately, when many regimes have been shown to be effective, treatment choice is more arbitrary and may depend on local protocols and patient factors. 
For nonsevere PCP and when treatment can be given orally and even on an out-patient basis, the preferred regimens are: oral TMP-SMX or oral clindamycin-primaquine. Patients who improve on intravenous therapy may be switched to an oral alternative if they are clinically improved. For those who fail to respond in spite of 5-7 days of first-line treatment, there is little to choose in terms of efficacy between second-line agents and choice is normally governed by individual patient factors such as tolerability and severity of PCP. ${ }^{17}$

\section{Bacterial respiratory infections}

Bacterial respiratory infections include pneumonia, tracheitis, and bronchitis. The causative organisms are broadly similar to those in non-HIV infected individuals. Streptococcus pneumoniae, Haemophilus influenzae, Mycoplasma pneumonia, and Chlamydia pneumoniae are the most commonly isolated bacteria. S. pneumoniae predominates, accounting for up to $40 \%$ of all bacterial pneumonia. Other less common causes of pneumonia (ie, Chlamydia psittaci, Legionella, and Staphlycoccus aureus) should also be considered and tested for with serological tests, urinary antigen testing, and microbiological culture where possible.

The risk of invasive pneumococcal disease (IPD) is also greater in $\mathrm{HIV}$-infected patients than the general population. ${ }^{18}$ This may be partially explained by a predisposition to pneumococcal nasopharyngeal colonization. In a longitudinal cohort of 260 mother-infant pairs in Zambia, half of who were HIV-seropositive, HIV infection was associated with an increased risk of colonization, particularly with pediatric serotypes. ${ }^{19}$ The treatment of IPD in the HIV setting needs to be prompt and aggressive as it carries a higher mortality risk in HIV-infected individuals and a higher risk of recurrence. ${ }^{20}$ As with non-HIV infected individuals, high dose penicillin forms the cornerstone of treatment.

Bacterial tracheitis and recurrent bronchitis, often associated with bronchiectasis, are also frequent in HIV-infected individuals. The isolated bacterial pathogens are similar to those causing pneumonia and include $S$. pneumoniae, $H$. influenzae, S. aureus, and P. aeruginosa.

Empiric treatment is generally dependent upon the clinical presentation and the degree of immunosuppression. In patients presenting with typical symptoms of bacterial pneumonia plus focal consolidation on chest radiograph, the initial antibiotic regimen will be directed at the most common community-acquired pathogens and will normally include a penicillin-based antibiotic such as amoxicillin or co-amoxiclav with a macrolide, ie, clarithromycin or erythromycin, as first-line treatment.
The decision to treat as an outpatient or inpatient will depend on the clinical status and the reliability of the individual. In general, the approach to treatment of community-acquired pneumonia is similar to that in the HIV-seronegative patient and there is very little specific evidence to guide the treatment in HIV-infected individuals. Clearly, as with other patients, treatment is based on history such as recent hospital admission, travel history and country of residence, taking into account data on local antimicrobial susceptibilities, concurrent immunomodulators, and the degree of immunosuppression. Clinicians should also take into account existent antibiotic prophylaxis (eg, TMP-SMX for PCP prophylaxis) when making decisions regarding administering empiric treatment for intercurrent bacterial infections. Naturally, the choice of antimicrobials will also depend on whether there are any features present to suggest a nonbacterial cause, ie, PCP, tuberculosis, or viral pneumonitis, when more specific treatment is indicated.

\section{Central nervous system disease}

Disease of the central nervous system (CNS) is a common occurrence in HIV and may present as a space occupying lesion, meningoencephalitis, or with an AIDS-dementia complex. The mode of presentation, in conjunction with brain imaging and examination of the cerebral spinal fluid (CSF) helps to establish the correct diagnosis. Here we discuss the common manifestations of nonmycobacterial CNS infection - cerebral toxoplasmosis and cryptococcal meningitis.

\section{Cerebral toxoplasmosis}

Toxoplasmosis usually manifests with multiple brain abscesses and is the most common cause of cerebral mass lesions in HIV-infected individuals worldwide. Primary infection often occurs early in life and symptomatic infection occurs in immunocompromised individuals due to reactivation of latent toxoplasma cysts. The most common clinical manifestation of toxoplasmosis in AIDS is a limited encephalitis associated with headache, fever, and focal neurology, although a more diffuse encephalitis with confusion and altered consciousness may also occur.

Due to the impracticality of obtaining brain tissue from most patients, treatment for toxoplasmosis is generally undertaken on the basis of positive toxoplasma serology, a compatible history, and mass lesions on brain imaging. An objective clinical response with improvement in brain imaging at 2 weeks is taken to be sufficient evidence for diagnosis, and first-line therapy is effective in approximately $90 \%$ of patients with cerebral toxoplasmosis. ${ }^{21}$ 


\section{Treatment phase}

First-line treatment for cerebral toxoplasmosis is a 6-week course of pyrimethamine, sulphadiazine, and folinic acid. Pyrimethamine is given as an initial $200 \mathrm{mg}$ loading dose, followed by weight-based therapy $(50 \mathrm{mg} /$ day if weight less than $60 \mathrm{~kg}$ or $75 \mathrm{mg} /$ day if weight more than $60 \mathrm{~kg}$ ). Pyrimethamine is only available in oral preparation and in the unconscious patient must be given via a nasogastric tube but is poorly absorbed, necessitating the initial loading dose. Sulphadiazine (15 mg/kg, four times daily) may be given either orally or intravenously, although the oral route is preferred due to its good bioavailability. Folinic acid (leucovorin) is given to prevent the hematological toxicities of pyrimethamine.

The evidence for the stated regime is derived from two randomized double-blind studies comparing pyrimethaminesulphadiazine with pyrimethamine-clindamycin. Dannemann et al (1992) and Katlama et al (1996) both reported equivalent treatment success although there seemed to be a survival benefit in the pyrimethamine-sulphadiazine arm. ${ }^{7,22}$ When the two studies were combined in a Cochrane review, mortality was comparable,${ }^{23}$ suggesting that both regimes are a reasonable option. Thus, for those who cannot tolerate sulphadiazine, substitution with clindamycin $(300 \mathrm{mg}$, four times daily) is an acceptable alternative, especially since it is reported to be better tolerated than sulphadiazine.

Other regimes that are used for treatment include atovaquone (1500 mg, twice daily) with either sulphadiazine or pyrimethamine or therapy with TMP-SMX alone. Evidence suggests that these regimens are effective, but the studies lack sufficient patient numbers to be well powered and there is less clinical experience with these regimens. ${ }^{24}$ An observational study of 83 patients in the French West Indies had an $85 \%$ treatment success rate with TMP-SMX alone, although they reported a relapse rate of $30 \%$ and a multicenter Italian study with 40 patients in the TMP-SMX arm showed similar findings compared to the standard first-line therapy. ${ }^{25,26}$ This is promising due to the fact that TMP-SMX is widely available and cheap compared to other regimes, but the high rate of relapse is worrying and makes its use as a first-line agent unfavorable. Other sulphadiazine-sparing regimes that have been used with success for the treatment of toxoplasmosis include clarithromycin, azithromycin, doxycycline, and dapsone combined with pyrimethamine-folinic acid, but the evidence is limited and currently they do not perform as well as either of the first or second line regimes.

The routine use of corticosteroids in toxoplasmosis is discouraged as without a tissue/microbiological diagnosis, they cloud the picture in what is effectively a therapeutic drug trial, since cerebral inflammation associated with primary CNS lymphoma and Mycobacterium tuberculosis will also respond to corticosteroids.

\section{Maintenance therapy}

After the initial 6-week treatment phase, secondary prophylaxis or maintenance therapy is continued. This is lifelong or until there is immune recovery, ie, CD4 $>200$ cells $/ \mu \mathrm{L}$ for more than 6 months. There are few specific trials to guide secondary prophylaxis however, but evidence from studies looking at prophylaxis for PCP have shown that TMP-SMX at $480-960 \mathrm{mg} /$ day is highly effective ${ }^{27}$ and that while dapsone $(50 \mathrm{mg} /$ day) plus pyrimethamine (50 $\mathrm{mg}$ weekly) is effective,${ }^{28}$ it should only be used in those who are intolerant to sulphadiazine due to the dual effect of TMP-SMX in prophylaxis against PCP pneumonia.

\section{Cryptococcosis}

Cryptococcosis is the most common systemic fungal infection in HIV-infected individuals and is associated with profound immunosuppression, occurring usually at $\mathrm{CD} 4<50$ cells $/ \mu \mathrm{L}$ and is universally fatal if untreated. It presents as a subacute meningitis or meningoencephalitis and is complicated by raised intracranial pressures in up to $75 \%$ of patients. In disseminated cryptococcosis, CNS symptoms may be absent, but pulmonary infection may occur with diffuse pulmonary infiltrates, nodules on chest radiograph, and symptoms including fever, cough, and dyspnea.

\section{Induction treatment}

Treatment is divided into two phases, induction and maintenance. Across the developed world, most would agree that gold-standard induction therapy combines intravenous liposomal amphotericin B (4 mg/kg/day) and flucytosine $(100 \mathrm{mg} / \mathrm{kg} /$ day) for at least 2 weeks. However, this treatment combination has many side effects, with amphotericin causing renal toxicity and flucytosine causing bone marrow suppression. If this regimen is not tolerated, and also in resource poor settings, where these drugs may not be available, good results have also been shown with oral fluconazole ( $400 \mathrm{mg} /$ day) both alone and with flucystosine.$^{29,30}$ There is also evidence to suggest that fluconazole may increase the fungicidal effect of flucytosine, enabling lower doses of flucytosine to be administered and improving tolerability, although this has yet to be translated into current practice. ${ }^{31}$

Amphotericin B has historically been the drug of choice for cryptococcosis but causes substantial renal toxicity at 
the normal dose (generally $0.7 \mathrm{mg} / \mathrm{kg} /$ day). There is much evidence now to support the use of liposomal amphotericin B, which has high rates of CNS penetration and can therefore be used at lower doses. This leads to better rates of CSF sterilization, significantly reduces the incidence of renal toxicity, and improves drug tolerability. ${ }^{32-34}$ This has now become the standard of care in much of the developed world, although may be hard to source in resource-poor countries where HIV infection is prevalent.

Initial data for the use of flucytosine with amphotericin B came from a study in the pre-HIV era. This showed that the addition of flucytosine was associated with fewer treatment failures or relapses than amphotericin alone in cryptococcal meningitis. ${ }^{35}$ Furthermore, Van de Horst et al in 1997 demonstrated that the addition of flucytosine to amphotericin does not improve early outcome but reduces the risk of relapse by improving rates of CSF sterilization. ${ }^{36} \mathrm{~A}$ more recent study of HIV-associated cryptococcal meningitis in a resource-poor setting has added weight to the evidence that the addition of flucytosine speeds the sterilization of CSF. Nussbaum et al compared fluconazole monotherapy with fluconazole plus flucytosine and found faster CSF sterilization and a reduced early and late mortality in the combination arm. ${ }^{37}$

Not all studies are easily compared however, as some of the earlier studies used lower doses of amphotericin B and were performed before the widespread availability of liposomal preparation. In addition, some studies that compared amphotericin and fluconazole used a lower dose of fluconazole ( $200 \mathrm{mg} /$ day) than is standard practice currently, so the difference needs interpreting with caution. There is no robust data to guide treatment if either of the first-line agents cannot be tolerated due to severe side effects. Clinicians may choose to simply omit the flucytosine and continue with amphotericin alone, or treat with flucytosine and fluconazole in combination if the amphotericin must be discontinued. Generally, oral fluconazole is a good alternative when resources are not available for the gold standard treatment as it may be given with or without flucytosine.

Regarding the newer azoles (ie, voriconazole or posiconazole), there are no controlled studies to support their use and given their high cost, they are normally only used anecdotally for cases when fluconazole cannot be tolerated or has failed. There is no role for the echinocandins (ie, caspofungin, micafungin) in the treatment or prophylaxis of cryptococcal meningitis as the organism is inherently resistant to the entire class of drugs.

The timing of initiation of ART in cryptococcal disease remains an area of controversy. A prospective Zimbabwean study of 54 patients compared early initiation of ART (within 72 hours) with delayed ART (after 10 weeks). It was terminated early due to a markedly increased mortality in the early initiation arm. ${ }^{38}$ However, the fact that they did not compare any time points in between 72 hours and 10 weeks is a major drawback as this would be when most clinicians would choose to initiate therapy. In addition, the study used fluconazole monotherapy ( $800 \mathrm{mg}$ ), which would not normally be the treatment of choice due to slower fungal CSF clearance. It also had a median follow-up time of only 27 days, which limits its robustness. It is clear that further information is required before being able to judge the optimum time for ART initiation in this context, however current guidance from the IDSA is to start between 2 and 10 weeks after initiation of an amphotericin-based regime. ${ }^{39}$

\section{Maintenance therapy}

Maintenance treatment follows directly on from the induction phase and is normally started after the 2-week treatment phase provided there has been a good clinical response, although some clinicians prefer to repeat the CSF examination and only switch to maintenance therapy once CSF culture is cryptococcus negative. This is a realistic option in developed countries where most of these patients are treated as inpatients and the resources are available for repeated CSF examinations, but in resource-poor countries, repeated lumbar punctures and CSF culture is often not feasible. In guiding maintenance therapy, a number of studies have shown that oral fluconazole ( $400 \mathrm{mg}$ /day) has been shown to be superior at preventing relapses than placebo, oral itraconazole, and weekly amphotericin B injections. ${ }^{40,41}$ Maintenance treatment is continued for a total of 10 weeks before patients are stepped down to secondary prophylaxis (fluconazole $200 \mathrm{mg}$ daily), which is continued lifelong or until sustained immune reconstitution occurs.

\section{Gastrointestinal disease}

The most common symptoms of gastrointestinal (GI) disease in HIV are weight loss and diarrhea. However, patients may present with a variety of symptoms including dysphagia, nausea, abdominal pain, and anorexia. The GI tract may be affected by a wide range of pathogens, from the types of bacterial gastroenteritides that are common in non HIV-infected individuals to opportunistic fungi, viruses, and parasites. Now that ART use is widespread, the incidence of many of these opportunistic gut infections has diminished enormously; however, in late presenters and those unresponsive to ART, they are still observed to cause chronic infection. 


\section{Acute bacterial gastroenteritis}

As with bacterial respiratory infection, bacterial gastroenteritis is many times more common in HIV-infected individuals at all CD4 counts, but is particularly associated with older age and lower CD4 counts. The most commonly isolated organisms are non-typhi Salmonella (especially Typhimurium and Enteritidis), Shigella and Campylobacter, and Clostridium difficile has also been described in one large US cohort as the most common cause of bacterial diarrhea in hospitalized patients with HIV. ${ }^{42,43}$ Clinical presentation can be one of three: 1) a self-limiting gastroenteritis; 2) a more severe and prolonged gastroenteritis associated with bloody diarrhea, fever, and weight loss; or 3) septicemia with or without a preceding GI illness. Salmonella, in particular, can cause a recurrent septicemia in immunosuppressed patients, which is prone to relapse and may be due to the $\mathrm{T}$ cell dysfunction seen at all CD4 counts. ${ }^{44.46}$

Clearly the mode of presentation determines the type and route of treatment given. Acute bacterial gastroenteritis in a patient with $\mathrm{CD}>200$ cells $/ \mu \mathrm{L}$ does not necessarily need antimicrobial treatment and may be self-limiting. However, in the context of immunosuppression, treatment is generally recommended.

Salmonella gastroenteritis in an immune competent individual does not normally require treatment, and there are no trials in the HIV population to guide the management of patients with CD4 $>200$ cells/ $\mu \mathrm{L}$. However, in an individual on ART with a well-preserved CD4 count, many clinicians would agree that it might be reasonable to withhold antibiotics. On the other hand, some clinicians would view the risk of bacteremia and relapse to be sufficiently high, even in immune-reconstituted HIV-infected individuals to warrant treatment. Treatment should always be guided by in vitro sensitivity testing, but first-line empiric treatment with an oral fluoroquinolone is usual, ie, ciprofloxacin $500 \mathrm{mg}$ twice daily. There is however, growing resistance to ciprofloxacin worldwide in both Salmonella sp. and Campylobacter sp. ${ }^{47,48}$ and alternatives include third generation cephalosporins, azithromycin, or TMP-SMX. ${ }^{49}$ It is therefore important that local resistance patterns and protocols guide the choice of first-line therapy. In the case of bacteremic patients, some clinicians advocate the addition of a second agent and this may be advised until strain sensitivities are known, but there is no current evidence to suggest that dual therapy rather than single agent therapy is associated with a better outcome.

The length of treatment for salmonellosis is poorly defined, but 5-7 days is generally adequate. Bacteremic patients are often treated for 2 weeks but there is little evidence on which to base this. Studies from the pre-ART era and from Africa have reported recurrent bacteremia in as many as $45 \%-47 \% .^{50,51}$ This has been illustrated to be due to recrudescence rather than re-infection and raises the question of whether long-term suppressive therapy is indicated in those with CD4 $<200$ cells $/ \mu \mathrm{L} .{ }^{52}$ However, in more recent years when it is rare not to be able to construct an effective ART regime, the risk of recurrence has not been shown to be reduced by long-term fluoroquinolone prophylaxis. ${ }^{48}$

Campylobacteriosis, as with salmonellosis, does not necessitate treatment if mild and in an immune competent host. However, in the immunocompromised and in those with persisting disease, antimicrobials are indicated. Fluoroquinolones have historically been the mainstay of treatment, but with emerging resistance patterns, macrolides such as azithromycin are being used effectively as first-line treatment. ${ }^{53}$

Shigella gastroenteritis may be mild and self-limiting, but there is good evidence that the use of antibiotics decreases the duration of symptoms and the risk of recurrence.$^{54}$ Treatment with a fluoroquinolone for 5 days is effective, as are TMPSMX or azithromycin. Specific treatment is guided by clinical response and local sensitivity patterns.

Treatment of $C$. difficile in HIV follows the same guidelines as for non-HIV infected individuals. The crux is to stop any antimicrobials predisposing to the infection and then to treat with metronidazole $400 \mathrm{mg}$ three times daily or vancomycin $125 \mathrm{mg}$ four times daily. A prospective study of $C$. difficile diarrhea has shown that HIV infection is not associated with worse outcomes or poorer response to standard treatment. ${ }^{55}$

\section{Cryptosporidiosis and microsporidiosis}

Cryptosporidia and microsporidia are protozoan parasites that cause an acute or subacute profuse, non-bloody, watery diarrhea, and are a common cause of chronic diarrhea in advanced HIV infection. ${ }^{56}$ Additional symptoms include nausea, vomiting, abdominal cramping, fever, and malabsorption. If the biliary tract is infected, a sclerosing cholangitis may arise, particularly in prolonged disease. The incidence of both diseases has declined dramatically with the introduction of ART. The most important treatment for both infections is the restoration of immune function and symptoms completely resolve without additional treatment once CD4 $>100$ cells $/ \mu \mathrm{L} .{ }^{57,58}$ ART is therefore the absolute cornerstone in the treatment of cryptosporidium and microsporidium. 


\section{Treatment of cryptosporidia}

There is little convincing evidence for the efficacy of antimicrobial therapy in the treatment of cryptosporidium. Multiple agents (nitazoxanide, paromomycin, spiramycin) have been shown in small randomized controlled trials to have some positive effect, but none have been shown to have a lasting effect without ART. ${ }^{59}$ Paromomycin is a nonabsorbable aminoglycoside, which is effective against cryptosporidia in animal models but there have been mixed data in HIVinfected adults and studies are limited by small numbers. ${ }^{60-62}$ A recent meta-analysis of its efficacy has shown no evidence for its use, although a small study of eleven patients showed a reduction in diarrheal frequency and oocyst shedding when given in combination with azithromycin. ${ }^{63,64}$

Nitazoxanide, a broad spectrum antiparasitic agent, has been approved for use in immunocompetent individuals but has not been shown to be superior to placebo in the severely immunocompromised in whom cryptosporidium is most problematic. ${ }^{65}$ There is little evidence to support its use and its widespread availability is questionable. In the absence of effective antimicrobials, supportive therapy with rehydration and electrolyte replacement is vital. Antimotility agents such as loperamide can provide symptomatic benefit.

\section{Treatment of microsporidia}

Microsporidia are ubiquitous organisms that are likely to be zoonotic and waterborne in origin. The most common microsporidia infecting the human gut are Enterocytozoon bieneusi and Encephalitozoon intestinalis. As with cryptosporidiosis, immune restoration with ART is the most important therapeutic consideration and in the context of profuse diarrhea, absorption of ART may need to be verified with therapeutic drug level monitoring.

There is no specific antimicrobial agent directed against E. bieneusi, although a single study showed that it may respond to oral fumagillin $(20 \mathrm{mg}$, three times daily for 14 days) ${ }^{66}$ However, fumagillin is not licensed for systemic therapy in the US or UK. Nitazoxanide, albendazole, and itraconazole have also been studied. Of these agents, albendazole (400 mg, twice daily for 21 days) has been shown to improve stool frequency and microsporidia clearance, although patient numbers in each study were small. .7,68 $^{67}$

\section{Candidiasis}

Candidal infection of the oropharynx is common and is a recognized surrogate marker of immunosuppression. ${ }^{69}$ Oral candida, diagnosed clinically by identification of the characteristic white plaques in the buccal mucosa or surface of the tongue, may be asymptomatic or symptomatic. Candidal infection of the esophagus, by contrast, is rarely asymptomatic and presents with fever, odynophagia, and retrosternal discomfort. It is unusual without coexisting oral candida and most physicians would treat empirically based on symptoms and the presence of oral candidiasis, only proceeding to endoscopy if symptoms do not rapidly improve with treatment. The most important differentials to rule out are herpes simplex virus or cytomegalovirus (CMV) esophagitis, which endoscopy with biopsy or brushings is able to do.

Treatment of oral candida is effective with both topical agents, ie, nystatin suspension or amphotericin lozenges or systemic treatment with an oral azole. However, topical treatment has been shown to be associated with slower clearance of the yeast and a higher rate of relapse. ${ }^{70}$ Thus, unless there is a specific contraindication to the use of an azole, most clinicians would favor systemic treatment in the immunocompromised patient.

Regarding choice of azole, fluconazole, itraconazole, and ketoconazole are the most commonly used systemic treatments and they all have activity against oropharyngeal candida. ${ }^{71-74}$ A number of well-designed studies have shown that fluconazole and oral suspension itraconazole are superior to ketoconazole and tablet formulation itraconazole. .1174,75 $^{7}$ This is related to the properties of the itraconazole and ketoconazole capsules, which require gastric acid in order to facilitate absorption. Advanced HIV is often associated with achlorhydria, which impairs the efficacy of these capsule agents. The use of oral solution itraconazole bypasses this mechanism and has been shown to have a better bioavailability than the capsule formulation. ${ }^{75} \mathrm{~A}$ very large prospective Italian study compared fluconazole with itraconazole for the treatment of candida esophagitis and found similar cure rates but a better short-term response with fluconazole. ${ }^{76}$ Fluconazole is therefore usually the first-line agent of choice at a dose of $50-100 \mathrm{mg} /$ day for 7-14 days, depending on clinical response.

Fluconazole-refractory candida should be tested for fluconazole resistance and a number of other antifungal drugs, such as the newer azoles and the echinocandins, may be considered for treatment in these cases. ${ }^{77}$ The echinocandins, such as caspofungin and micafungin, have been shown to be comparable to fluconazole for the treatment of esophageal candidiasis although some may be associated with a higher relapse rate. ${ }^{78-80}$ Direct comparison between studies is however limited by the differing doses of fluconazole used in the various studies. However, a small study that looked at the efficacy of caspofungin against fluconazole-resistant 
esophageal candidiasis found that it was associated with a clinical response in $79 \%$ of patients that had a reduced susceptibility to fluconazole, making it a very good secondline option. ${ }^{81}$ The newer azoles, such as voriconazole and posiconazole, have also been shown to have activity against fluconazole-resistant strains. ${ }^{82,83}$ There is no role for primary or secondary prophylaxis in candidiasis due to the rapid emergence of resistant strains. ${ }^{84}$

In summary, fluconazole remains the usual first-line agent for both oral and esophageal canididasis, although topical agents may also be used with good effect in oral candidiasis. In the event of fluconazole-refractory disease, second-line treatment is successful with either an echinocandin or one of the newer azole agents.

\section{Multisystem or disseminated infections Atypical mycobacteria}

The most clinically important atypical mycobacterial infection is Mycobacterium avium intracellulare complex (MAC), which causes disseminated disease and presents with systemic features of infection in individuals with CD $4<50$ cells $/ \mu \mathrm{L}$. Commonly infected sites include the lungs, bone marrow, liver, and lymph nodes with mycobacteria often grown from blood culture and isolated from these normally sterile sites. Historically, treatment of MAC has been difficult and prolonged, but with the concomitant use of ART, treatment outcomes are much improved. Again, many studies predate the ART era, which has dramatically reduced the incidence of MAC as well as shifting its clinical presentation to include more focal disease and a well-recognized IRIS phenomenon.

Macrolide therapy, in addition to some of the traditional antituberculous drugs, forms the cornerstone of MAC treatment and randomized clinical trials with macrolide containing regimes have better outcomes than nonmacrolide containing regimens. ${ }^{56}$ A number of studies have compared clarithromycin with azithromycin and shown consistently that clarithromycin is associated with more rapid resolution of bacteremia. ${ }^{85,86}$ Azithromycin (500-600 mg daily) however, is better tolerated than clarithromycin and is a legitimate second-line option if drug-drug interactions or intolerance preclude the use of clarithromycin. ${ }^{87}$ Macrolide monotherapy is associated with drug resistance ${ }^{88}$ therefore a second and/or third agent is required. Most clinicians would agree that ethambutol is the second agent of choice as more than any other drug, it has been shown to be associated with reduced incidence of drug resistance and clinical relapse. ${ }^{60-62} \mathrm{~A}$ study that compared ethambutol to rifabutin as the second agent with clarithromycin showed no difference in bacteremia resolution rates but higher rates of relapse in the rifabutin arm. ${ }^{89}$

ART forms a vital component of the treatment of atypical mycobacterial infection. In light of this and on the basis of current evidence, ${ }^{2}$ normal practice is to start ART within 2 weeks of antimycobacterial therapy and if this is the case, it can be argued that a third agent is not required. In patients for whom it is not possible to construct an effective ART regime, or who have had a poor immune response to treatment, the addition of a third antimycobacterial agent may be warranted and this is usually rifabutin. Rifabutin has been shown to protect against drug-resistance, which may become more of a concern if an individual will need prolonged treatment, and in some studies has been associated with improved survival. ${ }^{89,90}$ However, the use of rifabutin complicates the construction of an ART regime due to its potent induction of the CYP 450 liver enzyme and many clinicians opt for dual therapy in conjunction with ART, omitting rifabutin. Evidence from the pre-ART era suggests that the addition of rifabutin improved microbiological clearance and survival rates; ${ }^{89,90}$ however, in the context of effective ART, rifabutin may no longer be a necessary component of MAC treatment. Other agents such as clofazamine and ciprofloxacin have also been compared, but have poorer clinical outcomes. ${ }^{91,92}$

Treatment failure is defined as lack of clinical response and persistent mycobacteremia after 4-8 weeks of treatment. MAC isolates should be tested for drug susceptibility to clarithromycin and azithromycin, however the predicative value of susceptibility testing for ethambutol and rifabutin has not been confirmed. ${ }^{93}$ Susceptibility testing should guide the construction of a new regime and using the same principles as treating drug-resistant Mycobacterium tuberculosis, should include at least two new drugs from different classes to which the isolate should be susceptible. These include rifabutin (if not previously used), a quinolone (ciprofloxacin, levofloxacin, or moxifloxacin), linezolid, or amikacin. ${ }^{94}$ Other third-line agents such as prothionamide or cycloserine have only been used anecdotally and their efficacy is unknown.

In cases of refractory MAC, where immune reconstitution has not occurred and combination antimycobacterial therapy has not been successful, there is a limited amount of emerging evidence to support the use of immune modulators. One small case series has reported that low-dose dexamethasone reduces symptoms such as fever and weight loss, but this has yet to be substantiated fully. ${ }^{95}$ Another small case series reported an increase in monocyte activity in the blood after the co-administration of granulocyte colony-stimulating 
factor (gCSF) with antimycobacterial therapy, but this was not associated with a significant decrease in mycobacteremia. ${ }^{96}$ Interferon gamma has also shown some mixed results. It has been shown to increase the phagocytosis of MAC by blood monocytes in an HIV-infected patient with advanced MAC, ${ }^{97}$ but although there are a few case reports of clinical and radiological improvement in non HIV-infected individuals, the case reports in HIV-infected individuals report little sustained effect. ${ }^{98}$

There are no randomized studies to guide the duration of MAC treatment. However, most evidence suggests that treatment may be discontinued once immune recovery has been achieved. In practice, this constitutes a clinical response to MAC treatment for at least 3 months and both HIV virological suppression and immune recovery with a CD4 $>100$ cells $/ \mu \mathrm{L}$ for more than 3 months. If these criteria have not been achieved then the best evidence suggests that treatment should be lifelong and overall prognosis is poor.

\section{CMV infection}

CMV disease is caused by reactivation of the latent virus that is usually acquired in childhood. It occurs in those with CD4 counts below 100 cells $/ \mu \mathrm{L}$, but predominates in individuals with CD $4<50$ cells $/ \mu \mathrm{L}$. The widespread use of ART has dramatically decreased its incidence and has led to the recognition of CMV IRIS as a distinct presentation during immune recovery. It is a multisystem infection causing endorgan disease in the eye, gut, lung, and CNS.

\section{CMV eye disease}

Retinitis is the most common clinical manifestation of CMV disease. It usually begins unilaterally, but without immune reconstitution or systemic treatment, bilateral disease ensues almost universally. Diagnosis is made clinically with dilated fundoscopy by an experienced ophthalmologist. Treatment is indicated for all new cases, progression of existing lesions and reactivation of old lesions and seeks to limit progression of the retinitis and to reduce the risk of sight loss due to both macular involvement and retinal detachment.

Treatment can either be given locally or systemically. Local treatment via intraocular implants or injections has the advantage of being able to deliver high doses of drug directly to the affected area and avoids the toxic side effects of systemic treatment; systemic treatment however provides necessary prophylaxis to the contralateral eye and most HIV physicians would consider it to be best practice unless there are specific contraindications to its use. Drug therapy may be individualized to some extent according to the location and severity of disease, the degree of immune suppression, the likelihood of overlapping drug toxicities, and the ability of a patient to adhere to treatment.

Treatment is divided into an induction period of 2-4 weeks and a maintenance phase of treatment at lower doses which is continued until immune reconstitution occurs.

\section{Induction treatment}

Antivirals such as oral valganciclovir (900 mg twice daily), intravenous ganciclovir ( $5 \mathrm{mg} / \mathrm{kg}$ twice daily), foscarnet (90 mg/kg twice daily), and cidofovir ( $5 \mathrm{mg} / \mathrm{kg}$ ) have all been shown to be effective and are all reasonable options in the treatment of CMV disease. ${ }^{99,100}$ However, data from the Valganciclovir Study Group sways opinion towards the use of oral valganciclovir, a prodrug of ganciclovir with excellent oral bioavailability, which has been demonstrated to maintain patients in remission for longer than intravenous ganciclovir and is therefore normally used as first-line treatment. ${ }^{101}$ Regarding local therapy, the use of a ganciclovir implant has been shown to be superior to treatment with intravenous ganciclovir alone and for this reason, if an individual presents with immediately sight-threatening lesions, ie, disease in zone 1 of the retina, many experts would advise local treatment in addition to systemic therapy. ${ }^{102}$ Other local treatments include intravitreal injections with ganciclovir, foscarnet, and fomivirsen but there is less robust evidence to support their use. ${ }^{103-105}$

\section{Maintenance treatment}

Due to the very high risk of relapse and progression, maintenance therapy or secondary prophylaxis is continued after the induction phase. Several regimes have been shown to be effective including oral valganciclovir $900 \mathrm{mg}$ daily, intravenous ganciclovir $5 \mathrm{mg} / \mathrm{kg}$ daily (or $6 \mathrm{mg} / \mathrm{kg}$ for 5 days a week), intravenous foscarnet $90 \mathrm{mg} / \mathrm{kg}$ daily (or $120 \mathrm{mg} / \mathrm{kg}$ for 5 days a week), and intravenous cidofovir $5 \mathrm{mg} / \mathrm{kg}$ fortnightly. Clearly, given the strong evidence supporting the use of oral valganciclovir, its ease of use compared to the other regimes makes it the most favorable option.

Maintenance treatment is continued until immune reconstitution occurs and ART should be started as soon as is feasible. There is no evidence to suggest that concurrent treatment with ART and anti-CMV therapy is associated with worse outcomes. In the absence of immune restitution, CMV relapse over time is inevitable. ${ }^{101}$

\section{Treatment of reactivation or progression}

Relapse may occur due to inadequate intraocular drug levels or due to drug resistance. ${ }^{106,107}$ Early relapse tends to be 
attributable to poor intraocular penetration of systemically administered drugs and is best treated with a local ganciclovir implant. This has been shown to control the eye disease for up to 6-8 months, by which time the implant will need replacement and immune recovery may well have occurred. ${ }^{108,109}$

Relapse that occurs early on in maintenance therapy and which does not affect zones 1 and 2 may be treated by re-induction with the same drug, ie, oral valganciclovir, and this is normally effective at controlling the retinitis, though normally for shorter amounts of time. Changing to a different drug does not produce superior outcomes unless drug resistance is suspected, while combination therapy is associated with a longer mean time to relapse but can be poorly tolerated due to drug toxicities. ${ }^{110}$

Significant drug resistance does occur in long-term treatment. Resistance rates are typically less than $10 \%$ in the first 3 months of treatment but rise to $25 \%-30 \%$ by 9 months of therapy. ${ }^{11-113}$ Later relapse is often due to drug resistance, which is conferred through the CMV UL97 and UL54 genes. If low-level resistance is detected, treatment with intraocular ganciclovir implant may be effective due to the higher concentration of drug administered. If high-level resistance occurs, then a treatment change is required.

\section{Extra-ocular CMV disease}

The diagnosis of CMV disease at any site outside the eye should prompt urgent referral for ophthalmologic examination to rule out concomitant CMV eye disease, which if undiagnosed can cause irreversible loss of sight. As with CMV retinitis, ART is vital to the long-term suppression of CMV and there is no data to suggest early ART initiation has any detrimental effect on acute CMV disease.

Gastrointestinal disease caused by CMV can affect any part of the gut, but colitis and esophagitis are the more common manifestations. Diagnosis is made by endoscopic examination, revealing characteristic ulceration of the gut mucosa, and histologic confirmation on biopsy which shows the classic intracytoplasmic "owl's eye" inclusions. Culture of CMV from biopsy samples has been shown to be unreliable as a diagnostic tool as immunosuppressed individuals may shed the virus asymptomatically without evidence of ulcerative disease. Intravenous ganciclovir and foscarnet are both effective treatments and normal treatment is for 14-28 days. If symptoms are not severe enough to alter absorption, oral valganciclovir may also be used effectively. Maintenance therapy is not recommended routinely for gut disease except in cases of relapse.
CMV disease of the nervous system accounts for less than $1 \%$ of clinical CMV disease but may present as an AIDS-dementia complex, a ventriculo-encephalitis, or a polyradiculitis. Diagnosis is made on the basis of a positive CSF CMV DNA polymerase chain reaction (PCR) and by characteristic patterns on gadolinium-enhanced magnetic resonance imaging. Treatment for neurologic disease has been somewhat extrapolated from the extensive studies carried out in CMV eye disease and there have been no prospective controlled studies for CNS CMV disease itself. Treatment with ganciclovir, foscarnet, cidofovir, and valganciclovir all decrease CSF CMV titers, but are not very efficient in stabilizing neurologic disease. A small study from the pre-ART era looked at combination treatment with ganciclovir and foscarnet, but found poor results. ${ }^{114} \mathrm{It}$ is likely that prompt treatment, early ART and combination therapy all play a role, but drug toxicity often limits the duration of combination therapy and optimum treatment is not known.

CMV pneumonitis is uncommon and presents with fever, dry cough, exertional dyspnea, and hypoxemia. Chest imaging typically shows bilateral interstitial infiltrates, but may show nodules, adenopathy, and even cavitation. The major challenge is in distinguishing between asymptomatic CMV viral shedding in the respiratory secretions and active CMV lung disease. A positive CMV culture from respiratory samples such as bronchoalveolar lavage has a low positive predictive value but a high negative predictive value. A biopsy sample, however, is more sensitive. Generally, a compatible clinical syndrome, radiology, and positive CMV biopsy in the absence of a more likely co-pathogen are required before treatment of CMV is warranted. Again, treatment with intravenous ganciclovir, foscarnet, or oral valganciclovir is effective.

Finally, there is controversy surrounding the use of CMV viremia detected by PCR as a diagnostic tool for CMV disease. ${ }^{115,116}$ No prospective studies currently exist looking at the levels of CMV viremia and future risk of CMV disease or the preventative value of preemptive treatment. However, studies are on-going, and until robust data shows a clinical benefit, treatment of CMV viremia in the absence of end-organ disease is not currently recommended.

\section{Conclusion}

The field of HIV medicine has grown with a remarkable speed and energy over the last 20 years. The advances that have been made regarding ART have radically changed the whole outlook of HIV for both the patients and the medical 
professionals concerned with their care. In contrast to the huge changes in drug treatment for HIV, advances in the treatment of the common OI have been relatively meager. However, optimal treatment of OI remains an important issue as patients continue to present with advanced HIV infection. While the main advance in recent years has been the use of early ART as an adjunct to antimicrobial therapy, there is ongoing controversy regarding the specific timing of ART initiation during an acute OI. In general, "the earlier the better" is the message from studies addressing the question, but there remains some uncertainty with TB, where TB-IRIS causes considerable morbidity, and with cryptococcal meningitis where early ART has been shown to increase mortality.

Treatment of OI continues to be plagued by three main issues. The first is that the side effect profile of many drugs often limits their use. The second is that the gold standard treatment for many OI relies on a period of intravenous therapy or a costly oral alternative and these are not an option for many in resource-poor countries due to financial and logistical constraints. Finally, antimicrobial resistance continues to emerge, especially in parts of the world that rely heavily on fluoroquinolones for the management of bacterial infections. So far, in spite of its prolific use in prophylaxis there is little evidence of widespread TMP-SMX-resistant PCP, but this would have detrimental consequences, especially in resource-poor countries. In conclusion, there remains an urgent need for continued research into the treatment of OI with the aim of shortening intravenous courses and improving oral therapies and finding new regimes with improved drug toxicity profiles.

\section{Disclosure}

No conflicts of interest were declared in relation to this paper.

\section{References}

1. Sabin CA, Schwenk A, Johnson MA, et al. Late diagnosis in the HAART era: proposed common definitions and associations with mortality. AIDS. 2010;24(5):723-727.

2. Abdool Karim SS, Naidoo K, Grobler A, et al. Timing of initiation of antiretroviral drugs during tuberculosis therapy. $N$ Engl J Med. 2010;362(8):697-706.

3. Zolopa A, Andersen J, Powderly W, et al. Early antiretroviral therapy reduces AIDS progression/death in individuals with acute opportunistic infections: a multicenter randomized strategy trial. PLoS One. 2009;4(5):e5575.

4. Blanc FX, Sok T, Laureillard D, et al. Significant enhancement in survival with early ( 2 weeks) vs late ( 8 weeks) initiation of highly active antiretroviral treatment (HAART) in severely immunosuppressed HIVinfected adults with newly diagnosed tuberculosis. 18th International AIDS Conference (IAC). July 18-23, 2010. Vienna, Austria.
5. Grant PM, Komarow L, Andersen J, et al. Risk factor analyses for immune reconstitution inflammatory syndrome in a randomized study of early vs deferred ART during an opportunistic infection. PLoS One. 2010;5(7):e11416.

6. Wharton JM, Coleman DL, Wofsy CB, et al. Trimethoprimsulfamethoxazole or pentamidine for Pneumocystis carinii pneumonia in the acquired immunodeficiency syndrome. A prospective randomized trial. Ann Intern Med. 1986;105(1):37-44.

7. Dannemann B, McCutchan JA, Israelski D, et al. Treatment of toxoplasmic encephalitis in patients with AIDS. A randomized trial comparing pyrimethamine plus clindamycin to pyrimethamine plus sulfadiazine. The California Collaborative Treatment Group. Ann Intern Med. 1992;116(1):33-43.

8. Helweg-Larsen J, Tsolaki AG, Miller RF, Lundgren B, Wakefield AE. Clusters of Pneumocystis carinii pneumonia: analysis of person-to-person transmission by genotyping. QJM. 1998;91(12): $813-820$.

9. Klein NC, Duncanson FP, Lenox TH, et al. Trimethoprimsulfamethoxazole versus pentamidine for Pneumocystis carinii pneumonia in AIDS patients: results of a large prospective randomized treatment trial. AIDS. 1992;6(3):301-305.

10. Safrin S, Finkelstein DM, Feinberg J, et al. Comparison of three regimens for treatment of mild to moderate Pneumocystis carinii pneumonia in patients with AIDS. A double-blind, randomized, trial of oral trimethoprim-sulfamethoxazole, dapsone-trimethoprim, and clindamycin-primaquine. ACTG 108 Study Group. Ann Intern Med. 1996;124(9):792-802.

11. Sattler FR, Cowan R, Nielsen DM, Ruskin J. Trimethoprimsulfamethoxazole compared with pentamidine for treatment of Pneumocystis carinii pneumonia in the acquired immunodeficiency syndrome. A prospective, noncrossover study. Ann Intern Med. 1988;109(4): 280-287.

12. US Department of Health and Human Services. Guidelines for the prevention and treatment of opportunistic infections in HIV-infected adults and adolescents, June 18 2008. Available at: http://aidsinfo.nih. gov/. Accessed March 3, 2011.

13. Bozzette SA, Sattler FR, Chiu J, et al. A controlled trial of early adjunctive treatment with corticosteroids for Pneumocystis carinii pneumonia in the acquired immunodeficiency syndrome. California Collaborative Treatment Group. N Engl J Med. 1990;323(21):1451-1457.

14. Montaner JS, Lawson LM, Levitt N, Belzberg A, Schechter MT, Ruedy J. Corticosteroids prevent early deterioration in patients with moderately severe Pneumocystis carinii pneumonia and the acquired immunodeficiency syndrome (AIDS). Ann Intern Med. 1990;113(1):14-20.

15. Consensus statement on the use of corticosteroids as adjunctive therapy for pneumocystis pneumonia in the acquired immunodeficiency syndrome. The National Institutes of Health-University of California Expert Panel for Corticosteroids as Adjunctive Therapy for Pneumocystis Pneumonia. N Engl J Med. 1990;323(21):1500-1504.

16. Hughes W, Leoung G, Kramer F, et al. Comparison of atovaquone (566C80) with trimethoprim-sulfamethoxazole to treat Pneumocystis carinii pneumonia in patients with AIDS. N Engl J Med. 1993;328(21): 1521-1527.

17. Smego RA Jr, Nagar S, Maloba B, Popara M. A meta-analysis of salvage therapy for Pneumocystis carinii pneumonia. Arch Intern Med. 2001;161(12):1529-1533.

18. Gilks CF, Ojoo SA, Ojoo JC, et al. Invasive pneumococcal disease in a cohort of predominantly HIV-1 infected female sex-workers in Nairobi, Kenya. Lancet. 1996;347(9003):718-723.

19. Gill CJ, Mwanakasale V, Fox MP, et al. Impact of human immunodeficiency virus infection on Streptococcus pneumoniae colonization and seroepidemiology among Zambian women. J Infect Dis. 2008;197(7):1000-1005.

20. Falco V, Fernandez de Sevilla T, Alegre J, et al. Bacterial pneumonia in HIV-infected patients: a prospective study of 68 episodes. Eur Respir J. 1994; $7(2): 235-239$. 
21. Luft BJ, Hafner R, Korzun AH, et al. Toxoplasmic encephalitis in patients with the acquired immunodeficiency syndrome. Members of the ACTG 077p/ANRS 009 Study Team. N Engl J Med. 1993;329(14): 995-1000.

22. Katlama C, De Wit S, O’Doherty E, Van Glabeke M, Clumeck N. Pyrimethamine-clindamycin vs pyrimethamine-sulfadiazine as acute and long-term therapy for toxoplasmic encephalitis in patients with AIDS. Clin Infect Dis. 1996;22(2):268-275.

23. Dedicoat M, Livesley N. Management of toxoplasmic encephalitis in HIV-infected adults (with an emphasis on resource-poor settings). Cochrane database Syst Rev. 2006;3:CD005420.

24. Chirgwin K, Hafner R, Leport C, et al. Randomized phase II trial of atovaquone with pyrimethamine or sulfadiazine for treatment of toxoplasmic encephalitis in patients with acquired immunodeficiency syndrome: ACTG 237/ANRS 039 Study. AIDS Clinical Trials Group 237/Agence Nationale de Recherche sur le SIDA, Essai 039. Clin Infect Dis. 2002;34(9):1243-1250.

25. Beraud G, Pierre-Francois S, Foltzer A, et al. Cotrimoxazole for treatment of cerebral toxoplasmosis: an observational cohort study during 1994-2006. Am J Trop Med Hyg. 2009;80(4):583-587.

26. Torre D, Casari S, Speranza F, et al. Randomized trial of trimethoprim-sulfamethoxazole versus pyrimethamine-sulfadiazine for therapy of toxoplasmic encephalitis in patients with AIDS. Italian Collaborative Study Group. Antimicrob Agents Chemother. 1998;42(6): 1346-1349.

27. Ribera E, Fernandez-Sola A, Juste C, et al. Comparison of high and low doses of trimethoprim-sulfamethoxazole for primary prevention of toxoplasmic encephalitis in human immunodeficiency virus-infected patients. Clin Infect Dis. 1999;29(6):1461-1466.

28. Girard PM, Landman R, Gaudebout C, et al. Dapsone-pyrimethamine compared with aerosolized pentamidine as primary prophylaxis against Pneumocystis carinii pneumonia and toxoplasmosis in HIV infection. The PRIO Study Group. N Eng J Med. 1993;328(21):1514-1520.

29. Larsen RA, Bozzette SA, Jones BE, et al. Fluconazole combined with flucytosine for treatment of cryptococcal meningitis in patients with AIDS. Clin Infect Dis. 1994;19(4):741-745.

30. Larsen RA, Leal MA, Chan LS. Fluconazole compared with amphotericin B plus flucytosine for cryptococcal meningitis in AIDS A randomized trial. Ann Intern Med. 1990;113(3):183-187.

31. Larsen RA, Bauer M, Weiner JM, et al. Effect of fluconazole on fungicidal activity of flucytosine in murine cryptococcal meningitis. Antimicrob Agents Chemother. 1996;40(9):2178-2182.

32. Bates DW, Su L, Yu DT, et al. Mortality and costs of acute renal failure associated with amphotericin B therapy. Clin Infect Dis. 2001;32(5):686-693.

33. Leenders AC, Reiss P, Portegies P, et al. Liposomal amphotericin B (AmBisome) compared with amphotericin B both followed by oral fluconazole in the treatment of AIDS-associated cryptococcal meningitis. AIDS. 1997;11(12):1463-1471.

34. Hamill RJ, Sobel JD, El-Sadr W, et al. Comparison of 2 doses of liposomal amphotericin B and conventional amphotericin B deoxycholate for treatment of AIDS-associated acute cryptococcal meningitis: a randomized, double-blind clinical trial of efficacy and safety. Clin Infect Dis. 2010;51(2):225-232.

35. Bennett JE, Dismukes WE, Duma RJ, et al. A comparison of amphotericin B alone and combined with flucytosine in the treatment of cryptoccal meningitis. N Engl J Med. 1979;301(3):126-131.

36. Van der Horst CM, Saag MS, Cloud GA, et al. Treatment of cryptococcal meningitis associated with the acquired immunodeficiency syndrome. National Institute of Allergy and Infectious Diseases Mycoses Study Group and AIDS Clinical Trials Group. N Engl J Med. 1997;337(1):15-21.

37. Nussbaum JC, Jackson A, Namarika D, et al. Combination flucytosine and high-dose fluconazole compared with fluconazole monotherapy for the treatment of cryptococcal meningitis: a randomized trial in Malawi. Clin Infect Dis. 2010;50(3):338-344.
38. Makadzange AT, Ndhlovu CE, Takarinda K, et al. Early versus delayed initiation of antiretroviral therapy for concurrent HIV infection and cryptococcal meningitis in sub-saharan Africa. Clin Infect Dis. 2010;50(11):1532-1538.

39. Perfect JR, Dismukes WE, Dromer F, et al. Clinical practice guidelines for the management of cryptococcal disease: 2010 update by the Infectious Diseases Society of America. Clin Infect Dis. 2010;50(3):291-322.

40. Bozzette SA, Larsen RA, Chiu J, et al. A placebo-controlled trial of maintenance therapy with fluconazole after treatment of cryptococcal meningitis in the acquired immunodeficiency syndrome. California Collaborative Treatment Group. N Engl J Med. 1991;324(9):580-584.

41. Powderly WG, Saag MS, Cloud GA, et al. A controlled trial of fluconazole or amphotericin B to prevent relapse of cryptococcal meningitis in patients with the acquired immunodeficiency syndrome. The NIAID AIDS Clinical Trials Group and Mycoses Study Group. N Engl J Med. 1992;326(12):793-798.

42. Sanchez TH, Brooks JT, Sullivan PS, et al. Bacterial diarrhea in persons with HIV infection, United States, 1992-2002. Clin Infect Dis. 2005;41(11):1621-1627.

43. Angulo FJ, Swerdlow DL. Bacterial enteric infections in persons infected with human immunodeficiency virus. Clin Infect Dis. 1995;21(Suppl 1):S84-S93.

44. Jacobs JL, Gold JW, Murray HW, Roberts RB, Armstrong D. Salmonella infections in patients with the acquired immunodeficiency syndrome. Ann Intern Med. 1985;102(2):186-188.

45. Tocalli L, Nardi G, Mammino A, Salvaggio A, Salvaggio L. Salmonellosis diagnosed by the laboratory of the 'L. Sacco' Hospital of Milan (Italy) in patients with HIV disease. Eur J Epidemiol. 1991;7(6):690-695.

46. Gilks CF, Brindle RJ, Otieno LS, et al. Life-threatening bacteraemia in HIV-1 seropositive adults admitted to hospital in Nairobi, Kenya. Lancet. 1990;336(8714):545-549.

47. Molina J, Casin I, Hausfater P, et al. Campylobacter infections in HIV-infected patients: clinical and bacteriological features. AIDS. 1995;9(8):881-885.

48. Hung CC, Hung MN, Hsueh PR, et al. Risk of recurrent nontyphoid Salmonella bacteremia in HIV-infected patients in the era of highly active antiretroviral therapy and an increasing trend of fluoroquinolone resistance. Clin Infect Dis. 2007;45(5):e60-e67.

49. Guerrant RL, Van Gilder T, Steiner TS, et al. Practice guidelines for the management of infectious diarrhea. Clin Infect Dis. 2001;32(3):331-351.

50. Gordon MA, Banda HT, Gondwe M, et al. Non-typhoidal salmonella bacteraemia among HIV-infected Malawian adults: high mortality and frequent recrudescence. AIDS. 2002;16(12):1633-1641.

51. Celum CL, Chaisson RE, Rutherford GW, Barnhart JL, Echenberg DF. Incidence of salmonellosis in patients with AIDS. J Infect Dis. 1987;156(6):998-1002.

52. Hung CC, Hsieh SM, Hsiao CF, Chen MY, Sheng WH. Risk of recurrent non-typhoid Salmonella bacteraemia after early discontinuation of ciprofloxacin as secondary prophylaxis in AIDS patients in the era of highly active antiretroviral therapy. AIDS. 2001;15(5):645-647.

53. Tribble DR, Sanders JW, Pang LW, et al. Traveler's diarrhea in Thailand: randomized, double-blind trial comparing single-dose and 3-day azithromycin-based regimens with a 3-day levofloxacin regimen. Clin Infect Dis. 2007;44(3):338-346.

54. Christopher PR, David KV, John SM, Sankarapandian V. Antibiotic therapy for Shigella dysentery. Cochrane Database Syst Rev. 2010;8:CD006784.

55. Lu SS, Schwartz JM, Simon DM, Brandt LJ. Clostridium difficileassociated diarrhea in patients with HIV positivity and AIDS: a prospective controlled study. Am J Gastroenterol. 1994;89(8):1226-1229.

56. Conlon CP, Pinching AJ, Perera CU, Moody A, Luo NP, Lucas SB. HIV-related enteropathy in Zambia: a clinical, microbiological, and histological study. Am J Trop Med Hyg. 1990;42(1):83-88. 
57. Carr A, Marriott D, Field A, Vasak E, Cooper DA. Treatment of HIV1-associated microsporidiosis and cryptosporidiosis with combination antiretroviral therapy. Lancet. 1998;351(9098):256-261.

58. Miao YM, Awad-El-Kariem FM, Franzen C, et al. Eradication of cryptosporidia and microsporidia following successful antiretroviral therapy. J Acquir Immune Defic Syndr. 2000;25(2):124-129.

59. Cabada MM, White AC Jr. Treatment of cryptosporidiosis: do we know what we think we know? Curr Opin Infect Dis. 2010;23(5):494-499.

60. Tzipori S, Rand W, Griffiths J, Widmer G, Crabb J. Evaluation of an animal model system for cryptosporidiosis: therapeutic efficacy of paromomycin and hyperimmune bovine colostrum-immunoglobulin. Clin Diagn Lab Immunol. 1994;1(4):450-463.

61. Hewitt RG, Yiannoutsos CT, Higgs ES, et al. Paromomycin: no more effective than placebo for treatment of cryptosporidiosis in patients with advanced human immunodeficiency virus infection. AIDS Clinical Trial Group. Clin Infect Dis. 2000;31(4):1084-1092.

62. White AC Jr, Chappell CL, Hayat CS, Kimball KT, Flanigan TP, Goodgame RW. Paromomycin for cryptosporidiosis in AIDS: a prospective, double-blind trial. $J$ Infect Dis. 1994;170(2):419-424.

63. Abubakar I, Aliyu SH, Arumugam C, Usman NK, Hunter PR. Treatment of cryptosporidiosis in immunocompromised individuals: systematic review and meta-analysis. Br J Clin Pharmacol. 2007;63(4):387-393.

64. Smith NH, Cron S, Valdez LM, Chappell CL, White AC Jr. Combination drug therapy for cryptosporidiosis in AIDS. $J$ Infect Dis. 1998;178(3):900-903.

65. Rossignol JF, Hidalgo H, Feregrino M, et al. A double- 'blind' placebocontrolled study of nitazoxanide in the treatment of cryptosporidial diarrhoea in AIDS patients in Mexico. Trans $R$ Soc Trop Med Hyg. 1998;92(6):663-666.

66. Molina JM, Tourneur M, Sarfati C, et al. Fumagillin treatment of intestinal microsporidiosis. N Engl J Med. 2002;346(25):1963-1969.

67. Molina JM, Chastang C, Goguel J, et al. Albendazole for treatment and prophylaxis of microsporidiosis due to Encephalitozoon intestinalis in patients with AIDS: a randomized double-blind controlled trial. J Infect Dis. 1998;177(5):1373-1377.

68. Dieterich DT, Lew EA, Kotler DP, Poles MA, Orenstein JM. Treatment with albendazole for intestinal disease due to Enterocytozoon bieneusi in patients with AIDS. $J$ Infect Dis. 1994;169(1):178-183.

69. Klein RS, Harris CA, Small CB, Moll B, Lesser M, Friedland GH. Oral candidiasis in high-risk patients as the initial manifestation of the acquired immunodeficiency syndrome. $N$ Engl J Med. 1984; 311(6):354-358.

70. Pons V, Greenspan D, Debruin M. Therapy for oropharyngeal candidiasis in HIV-infected patients: a randomized, prospective multicenter study of oral fluconazole versus clotrimazole troches. The Multicenter Study Group. J Acquir Immune Defic Syndr. 1993;6(12):1311-1316.

71. De Wit S, Weerts D, Goossens H, Clumeck N. Comparison of fluconazole and ketoconazole for oropharyngeal candidiasis in AIDS. Lancet. 1989;1(8641):746-748.

72. Smith DE, Midgley J, Allan M, Connolly GM, Gazzard BG. Itraconazole versus ketaconazole in the treatment of oral and oesophageal candidosis in patients infected with HIV. AIDS. 1991;5(11):1367-1371.

73. Meunier F, Aoun M, Gerard M. Therapy for oropharyngeal candidiasis in the immunocompromised host: a randomized double-blind study of fluconazole vs ketoconazole. Rev Infect Dis. 1990;12(Suppl 3): S364-S368.

74. Graybill JR, Vazquez J, Darouiche RO, et al. Randomized trial of itraconazole oral solution for oropharyngeal candidiasis in HIV/AIDS patients. Am J Med. 1998;104(1):33-39.

75. Cartledge JD, Midgely J, Gazzard BG. Itraconazole solution: higher serum drug concentrations and better clinical response rates than the capsule formulation in acquired immunodeficiency syndrome patients with candidosis. J Clin Pathol. 1997;50(6):477-480.

76. Barbaro G, Barbarini G, Calderon W, Grisorio B, Alcini P, Di Lorenzo G. Fluconazole versus itraconazole for candida esophagitis in acquired immunodeficiency syndrome. Candida Esophagitis. Gastroenterology. 1996;111(5):1169-1177.
77. Metcalf SC, Dockrell DH. Improved outcomes associated with advances in therapy for invasive fungal infections in immunocompromised hosts. J Infect. 2007;55(4):287-299.

78. Villanueva A, Gotuzzo E, Arathoon EG, et al. A randomized doubleblind study of caspofungin versus fluconazole for the treatment of esophageal candidiasis. Am J Med. 2002;113(4):294-299.

79. Krause DS, Simjee AE, van Rensburg C, et al. A randomized, doubleblind trial of anidulafungin versus fluconazole for the treatment of esophageal candidiasis. Clin Infect Dis. 2004;39(6):770-775.

80. De Wet N, Llanos-Cuentas A, Suleiman J, et al. A randomized, doubleblind, parallel-group, dose-response study of micafungin compared with fluconazole for the treatment of esophageal candidiasis in HIV-positive patients. Clin Infect Dis. 2004;39(6):842-849.

81. Kartsonis N, DiNubile MJ, Bartizal K, Hicks PS, Ryan D, Sable CA. Efficacy of caspofungin in the treatment of esophageal candidiasis resistant to fluconazole. J Acquir Immune Defic Syndr. 2002;31(2): 183-187.

82. Skiest DJ, Vazquez JA, Anstead GM, et al. Posaconazole for the treatment of azole-refractory oropharyngeal and esophageal candidiasis in subjects with HIV infection. Clin Infect Dis. 2007;44(4):607-614.

83. Perfect JR, Marr KA, Walsh TJ, et al. Voriconazole treatment for lesscommon, emerging, or refractory fungal infections. Clin Infect Dis. 2003;36(9):1122-1131.

84. Wilcox CM, Alexander LN, Clark WS, Thompson SE 3rd. Fluconazole compared with endoscopy for human immunodeficiency virusinfected patients with esophageal symptoms. Gastroenterology. 1996;110(6):1803-1809.

85. Dunne M, Fessel J, Kumar P, et al. A randomized, double-blind trial comparing azithromycin and clarithromycin in the treatment of disseminated Mycobacterium avium infection in patients with human immunodeficiency virus. Clin Infect Dis. 2000;31(5): $1245-1252$.

86. Ward TT, Rimland D, Kauffman C, Huycke M, Evans TG, Heifets L. Randomized, open-label trial of azithromycin plus ethambutol vs clarithromycin plus ethambutol as therapy for Mycobacterium avium complex bacteremia in patients with human immunodeficiency virus infection. Veterans Affairs HIV Research Consortium. Clin Infect Dis. 1998;27(5):1278-1285.

87. Koletar SL, Berry AJ, Cynamon MH, et al. Azithromycin as treatment for disseminated Mycobacterium avium complex in AIDS patients. Antimicrob Agents Chemother. 1999;43(12):2869-2872.

88. Chaisson RE, Benson CA, Dube MP, et al. Clarithromycin therapy for bacteremic Mycobacterium avium complex disease. A randomized, double-blind, dose-ranging study in patients with AIDS. AIDS Clinical Trials Group Protocol 157 Study Team. Ann Intern Med. 1994;121(12): 905-911.

89. Benson CA, Williams PL, Currier JS, et al. A prospective, randomized trial examining the efficacy and safety of clarithromycin in combination with ethambutol, rifabutin, or both for the treatment of disseminated Mycobacterium avium complex disease in persons with acquired immunodeficiency syndrome. Clin Infect Dis. 2003;37(9):1234-1243.

90. Gordin FM, Sullam PM, Shafran SD, et al. A randomized, placebocontrolled study of rifabutin added to a regimen of clarithromycin and ethambutol for treatment of disseminated infection with Mycobacterium avium complex. Clin Infect Dis. 1999;28(5):1080-1085.

91. Chaisson RE, Keiser P, Pierce M, et al. Clarithromycin and ethambutol with or without clofazimine for the treatment of bacteremic Mycobacterium avium complex disease in patients with HIV infection. AIDS. 1997;11(3):311-317.

92. Shafran SD, Singer J, Zarowny DP, et al. A comparison of two regimens for the treatment of Mycobacterium avium complex bacteremia in AIDS: rifabutin, ethambutol, and clarithromycin versus rifampin, ethambutol, clofazimine, and ciprofloxacin. Canadian HIV Trials Network Protocol 010 Study Group. N Engl J Med. 1996;335(6):377-383.

93. Heifets L, Mor N, Vanderkolk J. Mycobacterium avium strains resistant to clarithromycin and azithromycin. Antimicrob Agents Chemother. 1993;37(11):2364-2370. 
94. Dube MP, Torriani FJ, See D, et al. Successful short-term suppression of clarithromycin-resistant Mycobacterium avium complex bacteremia in AIDS. California Collaborative Treatment Group. Clin Infect Dis. 1999;28(1):136-138.

95. Wormser GP, Horowitz H, Dworkin B. Low-dose dexamethasone as adjunctive therapy for disseminated Mycobacterium avium complex infections in AIDS patients. Antimicrob Agents Chemother. 1994;38(9):2215-2217.

96. Kemper CA, Bermudez LE, Deresinski SC. Immunomodulatory treatment of Mycobacterium avium complex bacteremia in patients with AIDS by use of recombinant granulocyte-macrophage colonystimulating factor. J Infect Dis. 1998;177(4):914-920.

97. Kedzierska K, Paukovics G, Handley A, et al. Interferon-gamma therapy activates human monocytes for enhanced phagocytosis of Mycobacterium avium complex in HIV-infected individuals. HIV Clin Trials. 2004;5(2):80-85.

98. Lauw FN, van Der Meer JT, de Metz J, Danner SA, van Der Poll T. No beneficial effect of interferon-gamma treatment in 2 human immunodeficiency virus-infected patients with Mycobacterium avium complex infection. Clin Infect Dis. 2001;32(4):e81-e82.

99. Foscarnet-Ganciclovir Cytomegalovirus Retinitis Trial. 4. Visual outcomes. Studies of Ocular Complications of AIDS Research Group in collaboration with the AIDS Clinical Trials Group. Ophthalmology. 1994;101(7):1250-1261.

100. The ganciclovir implant plus oral ganciclovir versus parenteral cidofovir for the treatment of cytomegalovirus retinitis in patients with acquired immunodeficiency syndrome: The Ganciclovir Cidofovir Cytomegalovirus Retinitis Trial. Am J Ophthalmol. 2001;131(4):457-467.

101. Martin DF, Sierra-Madero J, Walmsley S, et al. A controlled trial of valganciclovir as induction therapy for cytomegalovirus retinitis. N Engl J Med. 2002;346(15):1119-1126.

102. Musch DC, Martin DF, Gordon JF, Davis MD, Kuppermann BD Treatment of cytomegalovirus retinitis with a sustained-release ganciclovir implant. The Ganciclovir Implant Study Group. $N$ Engl J Med. 1997;337(2):83-90.

103. Tognon MS, Turrini B, Masiero G, et al. Intravitreal and systemic foscarnet in the treatment of AIDS-related CMV retinitis. Eur $J$ Ophthalmol. 1996;6(2):179-182.

104. Cochereau I, Belayachi N, Diraison C, et al. Treatment of CMV retinitis with intravitreal foscarnet. J Fr Ophthalmol. 1998;21(10):723-726.

105. Velez G, Roy CE, Whitcup SM, Chan CC, Robinson MR. High-dose intravitreal ganciclovir and foscarnet for cytomegalovirus retinitis. Am J Ophthalmol. 2001;131(3):396-397.
106. Kuppermann BD, Quiceno JI, Flores-Aguilar M, et al. Intravitreal ganciclovir concentration after intravenous administration in AIDS patients with cytomegalovirus retinitis: implications for therapy. J Infect Dis. 1993;168(6):1506-1509.

107. Arevalo JF, Gonzalez C, Capparelli EV, et al. Intravitreous and plasma concentrations of ganciclovir and foscarnet after intravenous therapy in patients with AIDS and cytomegalovirus retinitis. $J$ Infect Dis. 1995;172(4):951-956.

108. Marx JL, Kapusta MA, Patel SS, et al. Use of the ganciclovir implant in the treatment of recurrent cytomegalovirus retinitis. Arch Ophthalmol. 1996;114(7):815-820.

109. Hatton MP, Duker JS, Reichel E, Morley MG, Puliafito CA. Treatment of relapsed cytomegalovirus retinitis with the sustained-release ganciclovir implant. Retina. 1998;18(1):50-55.

110. Combination foscarnet and ganciclovir therapy vs monotherapy for the treatment of relapsed cytomegalovirus retinitis in patients with AIDS. The Cytomegalovirus Retreatment Trial. The Studies of Ocular Complications of AIDS Research Group in Collaboration with the AIDS Clinical Trials Group. Arch Ophthalmol. 1996;114(1) 23-33.

111. Jabs DA, Enger C, Dunn JP, Forman M. Cytomegalovirus retinitis and viral resistance: ganciclovir resistance. CMV Retinitis and Viral Resistance Study Group. J Infect Dis. 1998;177(3):770-773.

112. Jabs DA, Martin BK, Forman MS, et al. Longitudinal observations on mutations conferring ganciclovir resistance in patients with acquired immunodeficiency syndrome and cytomegalovirus retinitis: The Cytomegalovirus and Viral Resistance Study Group Report Number 8 . Am J Ophthalmol. 2001;132(5):700-710.

113. Jabs DA, Enger C, Forman M, Dunn JP. Incidence of foscarnet resistance and cidofovir resistance in patients treated for cytomegalovirus retinitis. The Cytomegalovirus Retinitis and Viral Resistance Study Group. Antimicrob Agents Chemother. 1998;42(9): $2240-2244$.

114. Anduze-Faris BM, Fillet AM, Gozlan J, et al. Induction and maintenance therapy of cytomegalovirus central nervous system infection in HIV-infected patients. AIDS. 2000;14(5):517-524.

115. Edwards SG, Grover D, Scott C, et al. Cytomegalovirus viral load testing of blood using quantitative polymerase chain reaction in acutely unwell HIV-1-positive patients lacks diagnostic utility. Int $J$ STD AIDS. 2007;18(5):321-323.

116. Dieterich DT, Rahmin M. Cytomegalovirus colitis in AIDS: presentation in 44 patients and a review of the literature. J Acquir Immune Defic Syndr. 1991;4(Suppl 1):S29-S35.
HIV/AIDS - Research and Palliative Care

\section{Publish your work in this journal}

HIV/AIDS - Research and Palliative Care is an international, peerreviewed open-access journal focusing on advances in research in HIV, its clinical progression and management options including antiviral treatment, palliative care and public healthcare policies to contro viral spread. The journal welcomes original research, basic science,

\section{Dovepress}

clinical \& epidemiological studies, reviews \& evaluations, expert opinion \& commentary, case reports \& extended reports. The manuscript management system is completely online and includes a very quick and fair peer-review system. Visit http://www.dovepress.com/ testimonials.php to read real quotes from published authors. 